\title{
Effects of a Care Workshop on Caring Behaviors as Measured by Patients and Patient Satisfaction
}

\author{
Hui-Shan Chan", Hui-Ying Chu' ${ }^{2}$, Hsiang Yen ${ }^{3}$, Li-Na Chou ${ }^{4}$ \\ ${ }^{1}$ Department of Applied Cosmetology, National Tainan Junior College of Nursing, Tainan, Taiwan \\ ${ }^{2}$ Department of Living Services Industry, Tainan University of Technology, Tainan, Taiwan \\ ${ }^{3}$ Department of Nursing, Chang Gung University of Science and Technology, Taoyuan, Taiwan \\ ${ }^{4}$ Department of Nursing, National Tainan Junior College of Nursing, Tainan, Taiwan \\ Email: "Inchou@mail.ntin.edu.tw
}

Received 17 January 2015; accepted 31 January 2015; published 4 February 2015

Copyright (C) 2015 by authors and Scientific Research Publishing Inc.

This work is licensed under the Creative Commons Attribution International License (CC BY).

http://creativecommons.org/licenses/by/4.0/

(c) (i) Open Access

\section{Abstract}

Caring is a central focus of nursing, and patient satisfaction is a critical indicator of nursing care quality. The aim of this study was to determine the effects that a care workshop exerted on nurse caring behaviors as perceived by patients and reflected by patient satisfaction. A quasi-experimental, pretest-posttest design without a control group was applied. The intervention consisted of several facets: 1) formal educational sessions twice a week for six weeks, 2) an angel-master mentorship activity, and 3) posts of exemplary caring behavior and stories. A demographic questionnaire, the Modified Nurse Caring Behaviors Inventory, and the Hartford Hospital Satisfaction Survey were used in this study. Descriptive statistics were analyzed to evaluate participant demographic characteristics. Paired $t$ tests were used to determine the effects of a care workshop on nurse caring behaviors as perceived by patients and reflected by patient satisfaction. Of the 236 patients enrolled, $49.6 \%$ were women and $57.2 \%$ were admitted to medical-surgical units. Female patients rated nurse caring behaviors higher and reported greater patient satisfaction than did male patients on the pretest $(p<0.05)$ and 6 -week posttest $(p<0.01)$. Patient with medical-surgical admissions rated nurse caring behaviors higher and reported greater patient satisfaction than patients with emergency admissions did on the pretest and 6 -week posttest $(p<0.01)$. Nurse caring behaviors and patient satisfaction were positively correlated on the pretest $(p<0.05)$ and 6-week posttest $(p<0.01)$. Patient satisfaction scores increased after the intervention compared with the pretest scores $(p=\mathbf{0 . 0 0 0})$. Caring educational programs for nurses are commonly regarded to be an essential strategy for promoting care knowledge and practice and improving patient satisfaction. According to the favorable results of this study, care workshops must be pro-

\footnotetext{
*Corresponding author.
}

How to cite this paper: Chan, H.-S., Chu, H.-Y., Yen, H. and Chou, L.-N. (2015) Effects of a Care Workshop on Caring Behaviors as Measured by Patients and Patient Satisfaction. Open Journal of Nursing, 5, 89-95. 
vides to nurses in other units or hospitals in future studies.

Keywords

Quasi-Experimental Design, Nurse Caring, Patient Satisfaction, Nursing Education, Quality of Care

\section{Introduction}

In the mid to late twentieth century, nursing care shifted from a mechanistic paradigm to a caring-healing paradigm in the healthcare system. Caring is essential for many reasons, and is considered crucial for nursing care quality [1]. The Taiwanese Nursing Association (2009) [2], which published a formal Code of Ethics for Nurses as a guide for Taiwanese nurses in their professional practice, identified caring as one of the most crucial professional values. Nurse caring behavior is a primary concern in high-quality nursing care and affects patient satisfaction outcomes. Because the number of patients being hospitalized for treatment has increased, the relationships between nurse caring behaviors and patient satisfaction have been explored [3] [4]. Wolf (2012) [5] searched 2709 references and found the quality of nursing care is associated with both nurse caring behavior and patient satisfaction, but few intervention studies have examined improving the caring behavior of nurses and the effect of caring behavior on patient satisfaction outcomes. Researchers must understand how to apply caring knowledge in practice after participating in a caring program to enable patients to feel cared for and to satisfy patient needs. However, intervention studies focusing on nurse caring behavior and its effect on patients' satisfaction are few in Taiwan. Thus, the purpose of this study was to determine the effectiveness of a care workshop in improving patient satisfaction in Taiwan.

Caring is an interpersonal human act that results in the satisfaction of human needs when demonstrated effectively [6]. In a high-quality healthcare environment, caring promotes health and individual growth and facilitates an assistance-trust relationship through which feelings can be expressed, perceived, and accepted [6]. Over the past decades, caring has been an emerging topic of interest in healthcare professions, particularly in nursing, because caring is considered an essential nursing characteristic and the focus of a holistic nursing process [6] [7]. Caring has been attributed to high-quality nursing care. In caring, therapeutic relationships, patients appreciate healthcare workers who consistently express care, and rarely forget the healthcare workers' spontaneous acts of kindness and caring. Research and literature relevant to nursing have focused on the relationship between patient perceptions of nursing care quality and have demonstrated that high-quality care is characterized by a relationship existing between the patient and a nurse who demonstrated caring behavior [8] [9]. Several studies have focused on the perception of critical nurse caring behaviors by acute or chronic care patients, indicating that nurse caring is a moral practice integral to nurse-patient interactions that enables patients to feel cared for [10] [11].

High-quality nursing care is dependent on patient perceptions of nurse caring behavior; the perceptions influence the outcomes of patient satisfaction [11]. Patient satisfaction with nursing care has emerged as a key quality indicator during the last decade [12] [13]. Recently, researchers have described a link between nurse caring behavior and patient satisfaction, demonstrating that nurse caring behavior is a positive predictive factor for patient satisfaction outcomes [3] [14]. Nurse caring or patient satisfaction can be enhanced through changes in care provision. In an experimental study, Yeakel and colleagues (2003) [15] examined the impact of patient satisfaction after nurses participated in a multifaceted staff intervention; the results showed that the patients admitted after the intervention judged nurses as more caring and reported greater satisfaction than did patients admitted before the intervention. Sheikhmooesi et al. (2013) [16] investigated the efficacy of a caring education program for nurses on inpatient satisfaction and found that the intervention program significantly improved inpatient satisfaction $(p<0.05)$.

The aim of the study was to determine the effectiveness of a care workshop in improving patient satisfaction. The null hypotheses stated that 1 ) there was no significant difference in the rating of nurse caring behaviors between patients surveyed before and patients surveyed after nurses participate in a care workshop, and 2) there was no significant difference in the satisfaction between patients surveyed before and patients surveyed after nurses participate in a care workshop. 


\section{Methods}

\subsection{Subjects}

This study used a convenience sample of 236 patients. A total of 284 patients were admitted to hospital general medical-surgical units and emergency rooms during the pre-intervention and post-intervention periods of the study. Of the 126 patients admitted during the pre-intervention period, 106 were enrolled; 20 were not enrolled (3 refused and 17 were unable to complete the questionnaire). Of the 158 patients admitted during the post-intervention period, 130 were enrolled; 28 were not enrolled ( 7 refused and 21 were unable to complete the questionnaire).

\subsection{Intervention}

A quasi-experimental, pretest-posttest design without a control group was used. The intervention consisted of three facets: 1) formal educational sessions, 2) an angel-master mentorship activity, and 3) posts of exemplary caring behaviors and stories at the hospital to inspire the hospital staff. The formal educational sessions were held twice a week for six month for 178 registered nurses and licensed practical nurses who work in medicalsurgical units and emergency rooms. The content of educational sessions included a description of the project and a series of workshops about caring. In the angel-master mentorship activity, each nurse (the "angel") secretly selected a colleague as a "master." The angel performed one anonymous caring act for the master daily to express care. For instance, the angel might leave a cheerful message on a slip of paper or prepare a cup of coffee for the busy master. Exemplary caring behaviors and stories of expressing and receiving care were shared by the nurses, and high-quality examples of caring behaviors and stories were voted on by the registered nurses and licensed practical nurses and posted in the waiting rooms.

\subsection{Instruments}

Demographic questionnaire. This researcher-developed instrument was used to gather information related to patient characteristics, including age, gender, unit of admission, and length of stay.

Modified Nurse Caring Behaviors Inventory. A subset of eight items from the Caring Behaviors Inventory by Hegedus (1999) [17] comprised the following: 1) the nurses treat me as an individual; 2) the nurses respect my rights; 3) the nurse are always honest with me; 4) the nurses provide soothing reassurance through their touch; 5) when I am fearful, the nurses try to relieve my fears; 6) the nurses make me feel important; 7) when I'm sad and cry, the nurses stay with me; and 8) the nurses comfort me by their silent presence. Each question was rated on a 3-point scale: 1 (not sure), 2 (agree), and 3 (strongly agree). Therefore, scores could range from 8 to 24. From a previous analysis of responses by 138 patients, the eight items were determined to have adequate internally consistent $(\alpha=0.886)$. Data from the 236 patients in the present study confirmed that the eight items have strong internal consistency $(\alpha=0.974)$.

Hartford Hospital Satisfaction Survey. The original instrument, used with permission from the authors [15], contained six items. The first four items were rated using a 5-point scale, ranging from 1 (never) to 5 (always). The fifth item, "my overall impression of the health care received," was rated from 1 (poor) to 5 (excellent). The sixth item, "I would recommend this hospital to my family and friends," was rated as 1 (definitely would not), 2 (probably would not), 3 (probably would), or 4 (definitely would). Therefore, satisfaction scores ranged from 6 to 29. The instrument reliability of the Hartford Hospital Satisfaction Survey had an overall internal consistency of $\alpha=0.860$ [15]. Data from the 236 patients in this study confirmed that the six items on the survey had strong internal consistency $(\alpha=0.879)$.

\subsection{Data Collection}

Data was collected in two phases. First, pretest data were collected before the intervention by using the demographic questionnaire, the Modified Nurse Caring Behaviors Inventory, and the Hartford Hospital Satisfaction Survey, all completed individually by participants. In the second phase, 6 weeks post intervention, posttest data were collected using the same instruments.

\subsection{Ethical Consideration}

This study was approved by the Human Trials Committee of a hospital in Southern Taiwan (SCMH_IRB No.: 
1001004). Interested and eligible participants were informed about the study and written consent was obtained before participants completed the survey. Participants were informed that they could withdraw from the study at any time without reason or penalty.

\subsection{Data Analysis}

The SPSS 17.0 software package (SPSS Inc., Chicago, IL, USA) was used for statistical analysis. Descriptive statistics (frequency distributions and percentages) were used to describe the participant demographic characteristics. Pearson's correlation coefficients were used to analyze the relationship between nursing caring behaviors and patient satisfaction. Paired $t$ tests were used to determine the effects of the care workshop according to the results from the Modified Nurse Caring Behaviors Inventory and the Hartford Hospital Satisfaction Survey. A significance level of 0.05 was used.

\section{Results}

\subsection{Participants Characteristics}

Of the patients enrolled, $49.6 \%$ were women and $57.2 \%$ were admitted to medical-surgical unites. Mean patient age was $53.8 \pm 16.0$ years and mean length of stay was $4.6 \pm 2.7$ days. Most (87.5\%) patients were discharged from the hospital (Table 1).

\subsection{Pre- to Post-Intervention Changes}

Female patients rated nurse caring behaviors higher and reported greater patient satisfaction than did male patients on the pretest $(p<0.05)$ and 6 -week posttest $(p<0.01)$. Patient with medical-surgical admissions rated nurse caring behaviors higher and reported greater patient satisfaction than patients with emergency admissions did on the pretest and 6-week posttest $(p<0.01)$. Nurse caring behaviors and patient satisfaction were positively correlated on the pretest $(r=0.514, p<0.05)$ and 6-week posttest $(r=0.873, p<0.01)$ (Table 2). Patients who rated nurse caring behaviors higher reported greater satisfaction; thus, there was positive correlation between nurse caring behaviors and patient satisfaction.

Nurse caring behaviors scores were $22.3 \pm 2.6$ on the pretest and $23.3 \pm 2.6$ on the posttest. Patient satisfaction scores were $26.3 \pm 3.4$ on the pretest and $27.4 \pm 3.4$ on the posttest. Posttest scores between nurse caring behaviors and patient satisfaction differed significantly. Nurse caring behaviors and patient satisfaction increased after the intervention compared with before the intervention. Patients admitted after the intervention perceived nurses to be more caring than did patients admitted before the intervention $(p=0.000)$. Increased patient satisfaction scores were observed after the intervention compared with the scores before the intervention ( $p$ $=0.000)$ (Table 3). These significant differences indicated that the care workshop improved patient ratings of nurse caring behaviors and patient satisfaction.

\section{Discussion}

This study investigated the effects of a care workshop on the ratings of nurse caring behaviors and patient satisfaction. Educational interventions for nurse are commonly viewed as a vital strategy for promoting nurse caring knowledge and behavior because nursing quality increases patient satisfaction. Nurse caring behaviors and patient satisfaction were positively correlated. Nurses who are more caring employ more patient-centered caring skills in clinical practice, and patients feel more cared for and satisfied with the nursing care [18]. Educational programs enhance nurses' caring knowledge and practical competence to ensure that patients are willing to receive treatment, thereby enhancing patient satisfaction [16] [19]. The results of this study revealed that the scores of nurse caring and patient satisfaction significantly increased between the pretest and the posttest. These findings indicate that the caring intervention effectively improved nurses' caring knowledge and practice and also enhanced patient satisfaction.

Patients with emergency admissions reported lower scores on nurse caring behaviors and patient satisfaction than did patients with medical-surgical admissions. Emergency rooms are workplaces with high workload and stress. Nurses cannot optimize their caring qualities in this working environment, and therefore, emergency patients do not perceive high-quality care and patient satisfaction is reduced. Furthermore, increased workload at 
Table 1. Participants characteristics $(\mathrm{N}=236)$.

\begin{tabular}{ccc}
\hline Characteristic & N & $\%$ \\
\hline Male & 119 & 50.4 \\
Female & 117 & 49.6 \\
Unit of admission & & \\
Medical-surgical unit & 135 & 57.2 \\
Emergency room & 101 & 42.8 \\
Age (years) (Mean, SD) & $(53.8,16.0)$ & \\
30 - 40 & 34 & 14.4 \\
41 - 50 & 86 & 36.4 \\
$>50$ & 116 & 49.2 \\
Length of stay (days) (Mean, SD) & $(4.6,2.7)$ & 41.5 \\
1 - 4 & 98 & 58.5 \\
$>4$ & 138 & \\
\hline
\end{tabular}

Table 2. Correlation coefficient among nurse caring behavior and patient satisfaction before and after a care workshop intervention $(\mathrm{N}=236)$.

\begin{tabular}{ccccc}
\hline Measure & Nurse caring behaviors & & & \\
& Pre-test & & 6-week post-test & \\
& $r$ & $p$ & $r$ & $p$ \\
Patient satisfaction & $0.514^{*}$ & 0.023 & $0.873^{* *}$ & 0.003 \\
${ }^{*} p<0.05,{ }^{* *} p<0.01$. & & & &
\end{tabular}

Table 3. Nurse Caring Behaviors and Patient satisfaction before and after a care workshop intervention $(\mathrm{N}=236)$.

\begin{tabular}{ccccc}
\hline Dependent variables & Groups & Mean $\pm \mathrm{SD}$ & $\mathrm{T}$ & $p$ \\
\hline Nurse caring behaviors & Pre-intervention & $22.3 \pm 2.6$ & 4.22 & 0.000 \\
& Post-intervention & $23.3 \pm 2.6$ & 5.28 & 0.000 \\
\multirow{2}{*}{ Patient satisfaction } & Pre-intervention & $26.3 \pm 3.4$ & 3.66 & 0.000 \\
& Post-intervention & $27.4 \pm 3.4$ & 6.50 & 0.000 \\
\hline
\end{tabular}

emergency department and the shortage of nurses may leave some patients without proper care; and emergency patients present with more severe physical conditions than do patients in medical-surgical departments. If nurses cannot immediately meet the needs of emergency patients, patients may complain about the quality of care, this may negatively affect the emergency patients' satisfaction and perception of caring behavior by nurses. The negative reactions indicated that seriously ill patients were likely to perceive less overall quality of care and satisfaction [20]. These findings correspond with other results indicating that patients with emergency admissions report lower scores for quality of care and satisfaction than do patients with planned admissions [21] and patient's perception of condition, patient satisfaction, and nurse caring were predictors of intent to return [22].

The satisfaction of female patients depends on whether they receive the continual care of medical personnel [23]; therefore, enhancing nurses' caring behaviors toward patients can positively affect female patient satisfaction. In this study, female patients rated nurse caring behaviors higher and reported greater satisfaction than did male patients. Such a result is consistent with the findings by Alhusban and Abualrub (2009) [24] and Davis and Duffy (1999) [25]. In addition, the results showed that female patients felt more cared for and had more pleasant hospitalization experiences than did male patients. Such a result is inconsistent with the findings of Foss (2002) 
[26], who determined that female patients felt the nursing staff was less caring than did male patients.

This study may be the first in Taiwan to indicate that both nurse caring and patient satisfaction can be influenced favorably by a care workshop intervention. This study adopted the angel-master mentorship activity as an intervention for nurses in Taiwan, who are less familiar with verbally expressing care and concern, to encourage them to learn from each other and bravely express concern for others. In addition, exemplary caring behaviors and stories were highly evaluated by hospital colleagues and inspired hospital administrators and medical personnel in other departments to value caring behaviors, which were another favorable results of this study. Thus, improving both caring knowledge and practice through continuing educations and mentorship programs is essential to foster nurses' caring abilities [27].

\section{Conclusion}

This study revealed that patient satisfaction with the care offered by healthcare professionals can be improved by training staff to develop patient-centered caring skills that can be implemented in clinical practice. According to the results of the study, we recommend that healthcare professionals are trained in patient-centered caring skills and that training is extended to the entire organization. Furthermore, we suggest that hospital administrative supervisors improve the high-workload environment in emergency rooms, thereby enabling emergency room nurses to provide high-quality health care for emergency patients in a work environment with sufficient human resources.

\section{Limitations}

Study limitations were apparent in the sampling and methodology. Regarding sampling, only patients who were admitted to two settings at one hospital in Southern Taiwan were involved in the study. Another possible limitation was that the level of nurse caring behavior and patient satisfaction was measured subjectively, which might create a reporting bias. Moreover, long-term follow-up after the intervention was not arranged because of limited personnel resources and finances; thus, the long-term efficacy of the intervention could not be interpreted.

\section{Acknowledgements}

The authors wish to express appreciation for the participants and Tainan Municipal Hospital in Taiwan.

\section{References}

[1] Tanking, J. (2010) Nurse Caring Behavior. The Kansas Nurse, 85, 3-5.

[2] Taiwan Nurses Association (2009) A Guideline of the Clinical Ladder System. http://www.twna.org.tw/frontend/un10_open/welcome.asp

[3] Rafii, F., Hajinezhad, M.E. and Haghani, H. (2008) Nurse Caring and Patient Satisfaction in Iran. International Journal for Human Caring, 12, 14-23.

[4] Schmock, B.N., Breckenridge, D.M. and Benedict, K. (2009) Effect of Sacred Environment on Surgical Patient Outcomes: A Pilot Study. International Journal for Human Caring, 13, 49-59.

[5] Wolf, Z.R. (2012) Systematic Review of Effect of a Caring Protocol Provided by Nursing Staff on Patient Satisfaction of Adult Hospitalization Patients. International Journal for Human Caring, 16, 58-70.

[6] Watson, J. (1979) Nursing: The Philosophy and Science of Caring. Little, Brown \& Company, Boston.

[7] Rhodes, M.K., Morris, A.H. and Lazenby, R.B. (2011) Nursing at Its Best: Competent and Caring. Online Journal of Issues in Nursing, 16, 1.

[8] Burhans, L.M. and Alligood, M.R. (2010) Quality Nursing Care in the Words of Nurses. Journal of Advanced Nursing, 66, 1689-1697. http://dx.doi.org/10.1111/j.1365-2648.2010.05344.x

[9] Izumi, S., Baggs, J.G. and Knafl, K.A. (2010) Quality Nursing Care for Hospitalization Patients with Advanced Illness: Concept Development. Research in Nursing \& Health, 33, 299-315. http://dx.doi.org/10.1002/nur.20391

[10] Baldursdottir, G. and Jonsdottir, H. (2002) The Important of Nurse Caring Behaviors as Perceived by Patients Receiving Care at an Emergency Department. Heart \& Lung, 31, 67-75. http://dx.doi.org/10.1067/mhl.2002.119835

[11] Zamanzadeh, V., Azimzadeh, R., Rahmani, A. and Valizadeh, L. (2010) Oncology Patients’ and Professional Nurses’ Perceptions of Important Nurse Caring Behaviors. BMC Nursing, 9, 10. http://dx.doi.org/10.1186/1472-6955-9-10 
[12] Attree, M. (2001) Patients’ and Relatives’ Experiences and Perspectives of “Good” and “Not So Good” Quality Care. Journal of Advanced Nursing, 33, 456-466. http://dx.doi.org/10.1046/j.1365-2648.2001.01689.x

[13] Tagney, J. (2008) A Caring Profession? British Journal of Cardiac Nursing, 3, 549. http://dx.doi.org/10.12968/bjca.2008.3.12.31799

[14] Wolf, Z.R., Miller, P.A. and Devine, M. (2003) Relationship between Nurse Caring and Patient Satisfaction in Patients Undergoing Invasive Cardiac Procedures. Medsurg Nursing, 12, 391-396.

[15] Yeakel, S., Maljanian, R., Bobannon, R.W. and Coulombe, K.H. (2003) Nurse Caring Behaviors and Patient Satisfaction: Improvement after a Multifaceted Staff Intervention. Journal of Nursing Administration, 33, 434-436. http://dx.doi.org/10.1097/00005110-200309000-00002

[16] Sheikhmoonesi, F., Zarghami, M., Tirgari, T. and Khalilian, A. (2013) Effcet of Transactional Analysis Education to Nurses on Patients' Satisfaction. European Psychiatry, 28, 1.

[17] Hegedus, K.S. (1999) Providers' and Consumers’ Perspective of Nurses' Caring Behaviours. Journal of Advanced Nursing, 30, 1090-1096. http://dx.doi.org/10.1046/j.1365-2648.1999.01198.x

[18] Palese, A., Tomietto, M., Suhonen, R., Efstathiou, G., Tsangari, H., Merkouris, A., et al. (2011) Surgical Patient Satisfaction as an Outcome of Nurses' Caring Behaviors: A Descriptive and Correlation Study in Six European Countries. Journal of Nursing Scholarship, 43, 341-350. http://dx.doi.org/10.1111/j.1547-5069.2011.01413.x

[19] Shrestha, S., Petrini, M. and Turale, S. (2013) Newborn Care in Nepal: The Effects of an Educational Intervention on Nurses’ Knowledge and Practice. International Nursing Review, 60, 205-212. http://dx.doi.org/10.1111/inr.12017

[20] Otani, K., Waterman, B. and Dunagan, W.C. (2012) Patient Satisfaction: How Patient Health Conditions Influence Their Satisfaction. Journal of Healthcare Management, 57, 276-292.

[21] Frojd, C., Swenne, C.L., Rubertsoon, C., Gunningberg, L. and Wadensten, B. (2011) Patient Information and Participation Still in Need of Improvement: Evaluation of Patients' Perception of Quality of Care. Journal of Nursing Management, 19, 226-236. http://dx.doi.org/10.1111/j.1365-2834.2010.01197.x

[22] Elder, R., Neal, C., Davis, B.A., Almes, E., Whitledge, L. and Littlepage, N. (2004) Patient Satisfaction with Triage Nursing in Rural Hospital Emergency Department. Journal of Nursing Care Quality, 19, 263-268. http://dx.doi.org/10.1097/00001786-200407000-00013

[23] Weisman, C.S., Rich, D.E., Rogers, J., Crawford, K.G., Grayson, C.E. and Henderson, J.T. (2000) Gender and Patient Satisfaction with Primary Care: Turning in to Women in Quality Measurement. Journal of Women's Health \& GenderBased Medicine, 9, 657-665. http://dx.doi.org/10.1089/15246090050118189

[24] Alhusban, M.A. and Abualrub, R.F. (2009) Patient Satisfaction with Nursing Care in Jordan. Journal of Nursing Management, 17, 749-758. http://dx.doi.org/10.1111/j.1365-2834.2008.00927.x

[25] Davis, B.A. and Duffy, E. (1999) Patient Satisfaction with Nursing Care in a Rural and an Urban Emergency Department. Australian Journal of Rural Health, 7, 97-103. http://dx.doi.org/10.1046/j.1440-1584.1999.00231.X

[26] Foss, C. (2002) Gender Bias in Nursing Care? Gender-Related Difference in Patient Satisfaction with Quality of Nursing Care. Scandinavian Journal of Caring Science, 16, 19-26. http://dx.doi.org/10.1046/j.1471-6712.2002.00045.X

[27] Faiman, B. (2011) Overview and Experience of a Nursing e-Mentorship Program. Clinical Journal of Oncology Nursing, 15, 418-423. http://dx.doi.org/10.1188/11.CJON.418-423 
Scientific Research Publishing (SCIRP) is one of the largest Open Access journal publishers. It is currently publishing more than 200 open access, online, peer-reviewed journals covering a wide range of academic disciplines. SCIRP serves the worldwide academic communities and contributes to the progress and application of science with its publication.

Other selected journals from SCIRP are listed as below. Submit your manuscript to us via either submit@scirp.org or Online Submission Portal.
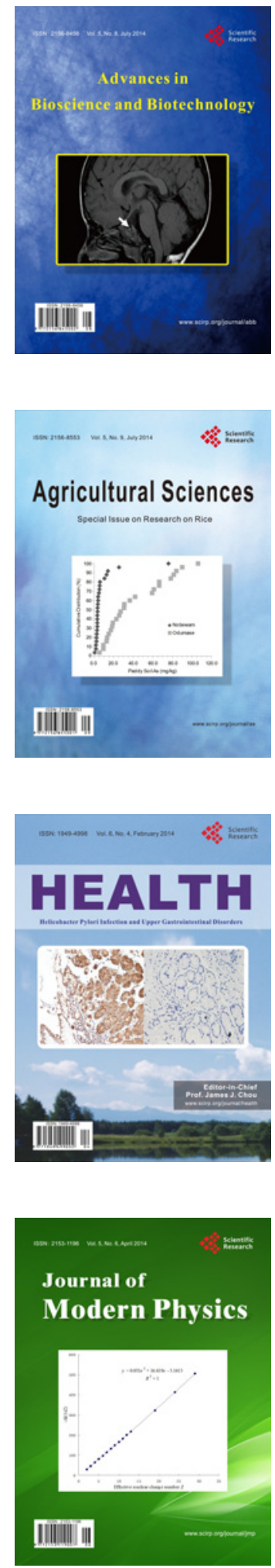
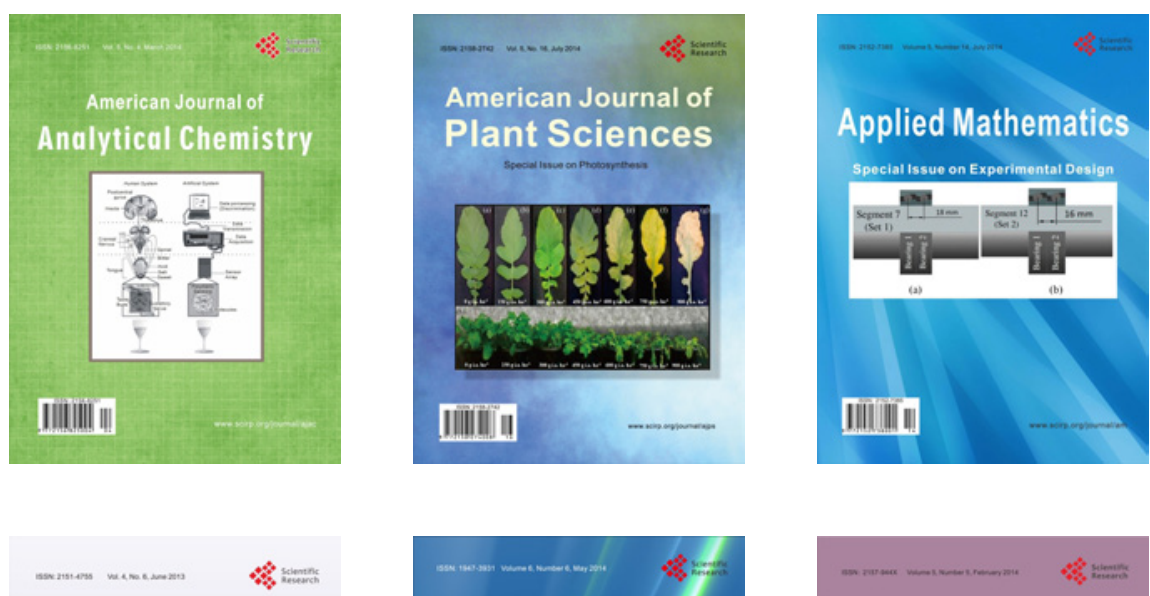

Creative Education
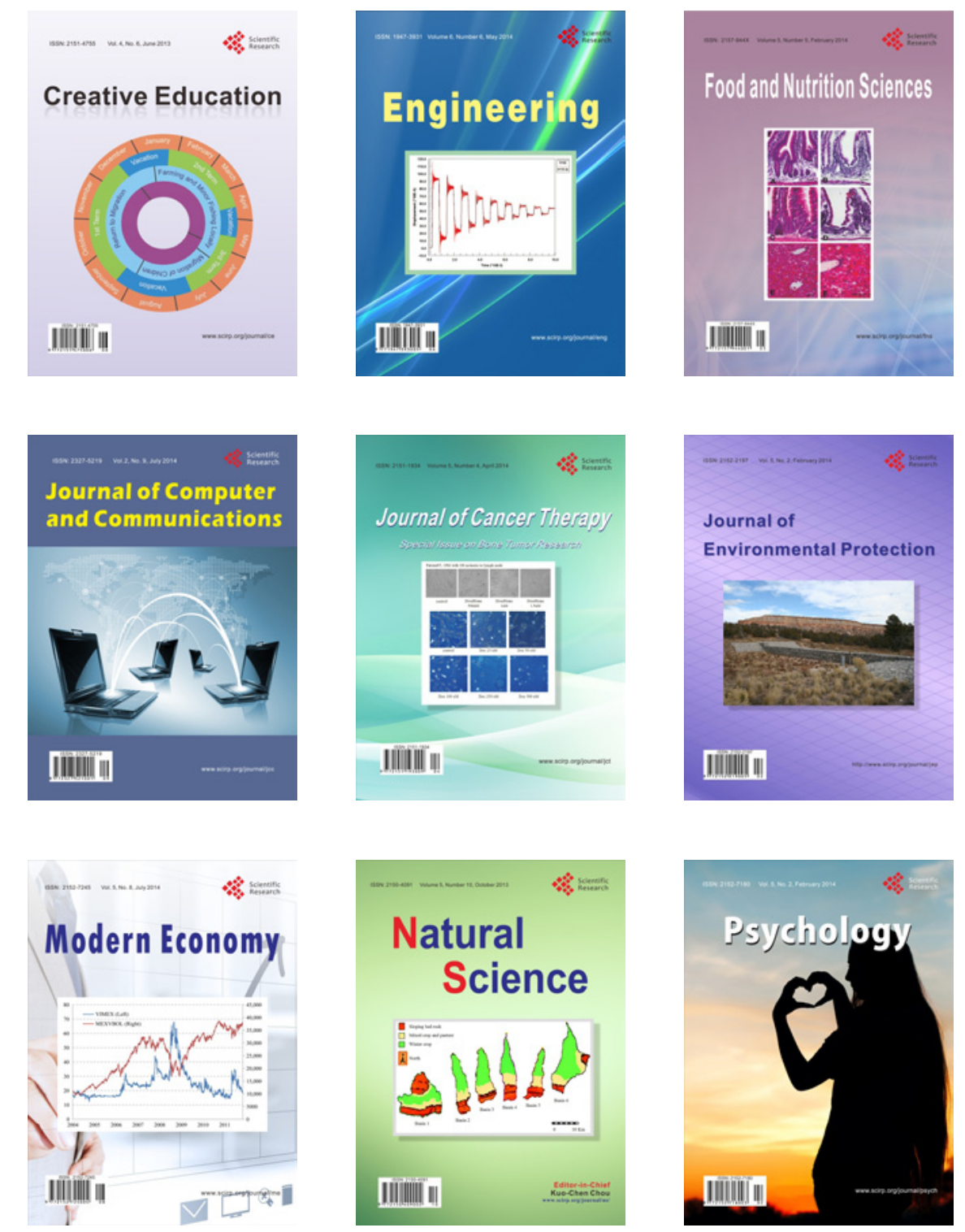